\title{
The role of ischemic preconditioning and pentoxifylline in intestinal ischemia/reperfusion injury of rats ${ }^{1}$
}

Teresinha Regina Ribeiro de Oliveira', Geraldo Ferreira de Oliveira", Ricardo Santos Simões"', Eduardo Hiroshi Tikazawa'v, Hugo Pequeno Monteirov, Djalma José Fagundes"v', Murched Omar TahavII

'PhD, Associate Professor, School of Health Sciences, Universidade Federal da Grande Dourados (UFGD), Dourados-MS, Brazil. Acquisition, analysis and interpretation of data; technical procedures; statistical analysis; manuscript preparation and writing.

"PhD, Associate Professor, School of Health Sciences, UFGD, Dourados-MS, Brazil. Acquisition of data, technical procedures, manuscript writing.

I'PhD, Department of Morfology and Genetic, Universidade de São Paulo (UNIFESP), Brazil. Histopathological examinations, interpretation and analysis of data.

IVMD, Resident, Public Health, Department Public Health, School of Medicine, Universidade Estadual de Campinas (UNICAMP), Brazil. Acquisition of data, technical procedures, manuscript preparation.

${ }^{V}$ PhD, Full Professor, Department of Biochemistry, UNIFESP, Sao Paulo -SP, Brazil. Analysis and interpretation of data, critical revision.

VIPhD, Full Professor, Division of Surgical Techniques and Experimental Surgery, Department of Surgery, UNIFESP, Sao Paulo-SP, Brazil. Analysis and interpretation of data, manuscript preparation, critical revision.

VIIPhD, Associate Professor, Division of Surgical Techniques and Experimental Surgery, Department of Surgery, UNIFESP, Sao Paulo-SP, Brazil. Conception and design of the study, critical revision, final approval.

\section{Abstract}

Purpose: To investigate the role of ischemic preconditioning (IPC) and pentoxifylline (PTX) in intestinal mucosa ischemia/reperfusion injury (IR).

Methods: Thirty rats were assigned to 5 groups $(\mathrm{N}=6)$ : $(\mathrm{CG})$ : no clamping of the superior mesenteric artery (90 min.); (IR-SS): saline + ischemia (30 min.) + reperfusion (60 min.); (IRPTX): PTX + ischemia (30min.) + reperfusion (60 min.); (IPC-IR-SS): $5 \mathrm{~min}$. of ischemia + 5 minutes of reperfusion (IPC) + saline + ischemia (30 min.) + reperfusion (60 min.); (IPC-IR-PTX ): 5 min. of ischemia +5 min. of reperfusion (IPC) + PTX +30 min. of I +60 minutes of $R$.

Results: The IR-PTX, IPC-IR-SS and IPC-IR-PTX groups had significantly lower scores of mucosa damage than the IR-SS group. IR-PTX group showed higher scores than the IPC-IR-PTX group, in accordance with the hypothesis of a favorable effect of IPC alone or in association with PTX. Additionally, IPC-IR-SS had a higher damage score than the IPC-IR-PTX. The villi height and crypt depth were similar in all groups. The villi height in the IR-SS was significantly lower. Conclusion: Ischemic preconditioning or pentoxifylline alone protect the intestinal mucosa from ischemia/reperfusion injury. However, they do not have a synergistic effect when applied together.

Key words: Ischemic Preconditioning. Pentoxifylline. Ischemia. Reperfusion Injury. Intestines. Rats. 


\section{Introduction}

Organs subjected to extended periods of ischemia (I) during major surgical procedures and reperfusion (R) following organ revascularization experience major oxidative stress conditions ${ }^{1-3}$. In the intestine, IR injuries are associated with a number of clinical conditions, which may include acute mesenteric ischemia, intestinal obstruction, and necrotizing colitis ${ }^{4,5}$. IR injury can also occur because of collapse of systemic circulation, as in hypovolemic and septic shock ${ }^{1}$. In addition, intestinal transplantation is also a situation in which the cascade of deleterious events associated with IR is relevant ${ }^{1,4,5}$.

Ischemic preconditioning (IPC) is an intrinsic process whereby repeated short episodes of ischemia protect the organ against a subsequent prolonged ischemic insult ${ }^{4-7}$. Several reports have described the effects of IPC on the reduction of apoptosis of epithelial cells $s^{4,5,7-9}$, structural protection ${ }^{4,10,11}$ and preservation of intestinal contractility ${ }^{10}$. In brief, the IPC stimulus, made up of cycles of brief ischemia and reperfusion, initiates production of a number of autacoids (such as acetylcholine, adenosine, bradykinin, endothelin, and opioids) ${ }^{11}$. These substances bind to their respective receptors on the plasma membrane to stimulate a number of signaling pathways that convey a protective signal to the mitochondria ${ }^{11,12}$, wherein a signaling of reactive oxygen species (ROS) occurs ${ }^{2,11,12}$. They activate protein kinases such as Akt, Erk1/2, protein kinase $C$, and tyrosine kinase, which provide the 'memory' in response to the subsequent and prolonged ischemia. This process allows the protective effect to last up to 2-3 hours. Despite intensive investigation, the actual mechanisms that mediate this protective effect remain incompletely understood, although a large number of signaling pathways underlying IPC have been identified in the past 30 years ${ }^{8,9,11,12}$.

On the other hand, the drugs pentoxifylline (PTX), a methylxanthine derivative of theobromine, has been proposed to decrease the deleterious effects of $\mathrm{IR}^{4,12-14}$. In clinical use for the 30 past years as well, it improves the rheological properties of the blood in numerous ways: decreasing plasma and whole blood viscosity, in large measure owing to a reduction in plasma fibrinogen; increasing erythrocyte distensibility and suppressing erythrocyte aggregation; diminishing platelet aggregation; and increasing blood filterability by suppressing neutrophil activation ${ }^{13}$.

The cumulative effect of a reduction in plasma viscosity, an increase in erythrocyte flexibility, and a suppression of neutrophil activation lead to an improvement in capillary blood flow, particularly in vascular beds downstream from arterial flux damage. Pentoxifylline also exerts anti-inflammatory and antioxidant effects. The antioxidant effects appear primarily attributable to decreased neutrophil activation, as activated neutrophils generate superoxide via NADPH oxidase. It lowers the plasma levels of proinflammatory cytokines such as tumor necrosis factor (TNF) $\alpha$, interleukin (IL) 1 and IL-6 $6^{12-14}$. The biologic pathways underlying these various effects remain rather murky.

Both procedures have common biological pathways and have not yet been investigated together. Therefore, we decided to investigate a possible additive protective effect through the use of IPC and PTX on intestinal mucosa using an intestinal IR model in rats.

\section{Methods}

Thirty male Wistar-EPM1 rats, 250 to 
300g, from the Center for the Development of Experimental Models for Medicine and Biology (CEDEME, Sao Paulo-SP, Brazil) remained under controlled temperature $\left(23^{\circ} \mathrm{C}\right), 50 \%$ humidity on, and light conditions with a 12-hour light/ dark. The animals were housed in a controlled environment at the Experimental Surgery Department of UNIFESP. The individual cages had dimensions of $40 \mathrm{~cm} \times 30 \mathrm{~cm} \times 25 \mathrm{~cm}$ and, which were covered with previously autoclaved wood shavings. Thad ad libitum access to water and balanced feed. All procedures involving anaesthesia and euthanasia were performed according to followed the guidelines of the National Council Animal Experimentation Control (SBCAL/COBEA - Brazil http://www. cobea.org.br/).

The animals were anesthetized by $80 \mathrm{mg} / \mathrm{kg}$ ketamine (Dopalen ${ }^{\circ}$, Sespo Indústria Comércio, Paulínea-SP, Brazil) and $10 \mathrm{mg} / \mathrm{kg}$ xylazine (Dopaser ${ }^{\circ}$, Herpate Callieri, JuatubaMG, Brazil) and were subjected to median laparotomy. Then, the superior mesenteric vessels were identified and clamped according to the group. The femoral vein was dissected and catheterized for infusions of drug $180 \mathrm{mg} /$ kg pentoxifylline, Sigma Aldrich, Sao Paulo-SP, Brazil) or $0.9 \%$ saline solution (in control group) 5 min before sustained ischemia. Afterwards, the animals were randomly distributed into five groups: the control group (CG) received anesthesia + laparotomy + no clamping of the superior mesenteric artery (time elapsed 90 minutes), the IR-SS group received saline +30 minutes of ischemia +60 minutes of reperfusion, the IR-PTX group received pentoxifylline +30 minutes of ischemia +60 minutes of reperfusion, the IPC-IR-SS group received five minutes of ischemia + five minutes of reperfusion (preconditioning) + saline +30 minutes of ischemia +60 minutes of reperfusion, and the IPC-IR-PTX group received five minutes of ischemia + five minutes of reperfusion (preconditioning) + pentoxifylline +30 minutes of ischemia +60 minutes of reperfusion.

At the end of the reperfusion period, one intestinal samples were collected $20 \mathrm{~cm}$ from the duodenum-jejunum flexure, opened longitudinally, gently washed in saline solution, and fixed in 10\% formaldehyde phosphatebuffered solution. The tissue samples were then processed for paraffin embedding and subsequently stained with hematoxylin and eosin (H.E.) for histological analysis. A Scanner (3D HISTECH ${ }^{\circ}$, Biogen, Sao Paulo$\mathrm{SP}$, Brazil) captured all images of histological slides. The images were analyzed by specific software (Case Viewer', 3D Histech Biogen, Sao Paulo-SP, Brazil; magnification x20, x40, $x 50)$. An independent pathologist, who had no knowledge of the origins of the experimental groups, undertook the histological study. We used the score for the damage based on the criteria of Chiu et al. ${ }^{15}$ : Grade 0 - mucosa without any histological alteration; Grade 1- intestinal villous well constituted, no cellular lysis and no inflammatory process, but subepithelial Grünhagen space present; Grade 2 - cellular lysis, presence of subepithelial Grünhagen space and distances among intestinal villous increased; Grade 3 - destruction of the tip portion of the villous, capillaries dilated and presence of inflammatory cells; Grade 4 structural destruction of intestinal villous, presence of inflammatory cells and necrotic material, hemorrhagic areas and loosening of the tip of the superficial intestinal mucosa villous; Grade 5 - destruction of the intestinal mucosa, no glandular structures preserved and presence of amorphous substance at the submucosal region. The data were expressed by mean \pm standard error $(x \pm$ se). The difference among the groups, a Kruskal-Wallis 
non-parametric test was followed by Bonferroni post hoc comparison test, and processed by statistic software (STAT 9.4, Stat Plus de Analyst Soft Inc.)

The quantitative measurement of the thickness of the intestinal wall was to switch the length of the villi from its outermost layer (serous) to its most prominent summit in the intestinal lumen. The standard of measurement for the crypts was the depth from the serosa to the bottom of the crypt. Ten fields were randomly chosen, for a total of 60 crypts and 60 villi, with the criteria of identifying villi in a visible field and a defined epithelium for reading measurements through the program (Case Viewer, 3D HISTECH), expressed in micrometers $(\mu \mathrm{m})$, The results were expressed as the means \pm standard error, and analyses of variance with repeated measures (ANOVA) followed Bonferroni post hoc comparison test. The level of rejection of the null hypothesis was set to $5 \%(P \leq 0.05)$. All calculations were performed using Stat software version $9.4^{\circ}$.

\section{- Results}

The histological sections in Figure 1 allowed us to verify the morphological changes in the intestinal mucosal layer in each of the five study groups.

The control group showed intact villi without disruption of enterocytes and no debris in the lumen (Figure1, A-GC). However, in the IR-SS group, in which intravenous injection of saline was followed by 30 minutes ischemia and 60 minutes of reperfusion, damage was evident (Figure 1, B-IR+SS). Destruction of the intestinal villi architecture, interstitial swelling, hemorrhagic foci and cellular debris in the intestinal lumen were observed. In the IR-PTX group (Figure $1 \mathrm{C}$ ), although the villi remained intact without cell lysis or inflammation, spaces (Grünhagen spaces) were present (Figure 1, C-IR+PTX). The IPC+IR+SS (Figure 1D) and IPC+IR+PTX (Figure 1E) groups showed similar findings, with cell lysis at the apex of the villi, interstitial vasodilatation, increased spacing among the villi and mild edema.

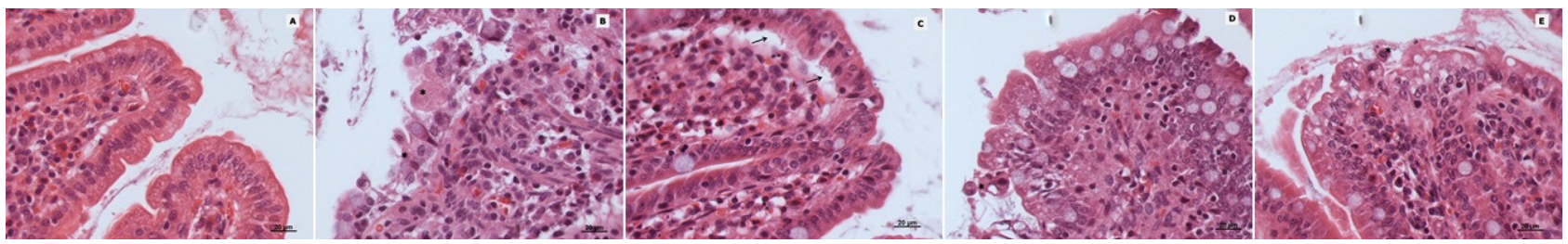

Figure 1 - Histological features of the rat intestinal mucosa. A, CG: total integrity of the epithelium. B, IR+SS group showed loss of the architecture of the intestinal villi and the presence of interstitial swelling $\left({ }^{*}\right)$. $\mathbf{C}$, IR+PTX group, had villi with normal structures and without cell lysis or inflammation, although subepithelial spaces (Grünhagen spaces) were present (arrows). The IPC+IR+SS (D) and IPC+IR+PTX (E) groups had similar findings with cell lysis at the apex of the villi $\left(^{*}\right)$ and interstitial vasodilatation (scale bar 20 $\mu \mathrm{m} \mathrm{H.E.} \mathrm{staining).}$

The qualitative histological evaluation was complemented by the lesion score based on the criteria of Chiu et al. ${ }^{15}$. Figure 2 shows the scores among the five groups and their statistical significance. The IR-PTX $(1.39 \pm 0.25)$, PCI-IR-SS (1.28 \pm 0.13$)$, and IPC-IR-PTX (1.06 \pm $0.13)$ groups showed significantly lower scores than the IR-SS $(3.67 \pm 0.42)$ group. These findings are consistent with a favorable effect of PTX and PCl alone or in combination. On the other hand, the IR-PTX group had higher scores than the IPC-IR-PTX groups, which is in accordance with the hypothesis of a favorable effect of IPC alone or in association with PTX. The IPC-IR-SS group had a higher damage score than the IPC-IR-PTX group. 


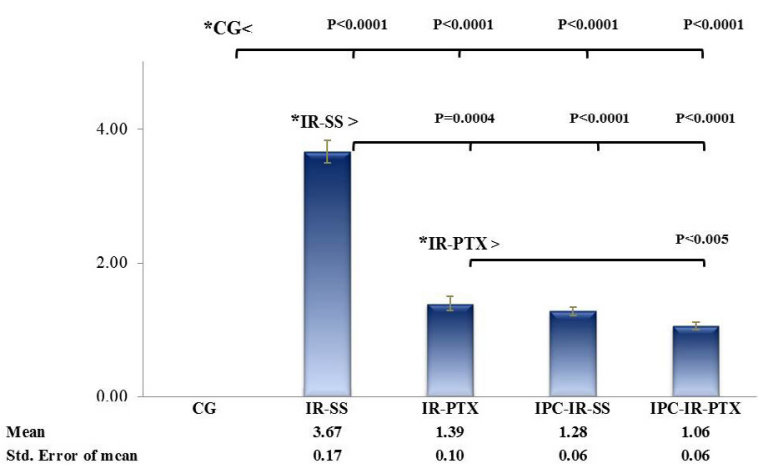

Figure 2 - Intestinal mucosal injury scores. Statistical analyses for all studied groups were performed using the Kruskal-Wallis $(H=25.45, P<0.0001)$ and pos hoc Bonferroni tests. $\left(^{*}\right)$ Statistically significant values: was represent in charts.

The villi height and crypt depth (Figures 3 and 4 , respectively) were standard in the control group. Figure 3 shows that the villi height in the IR-SS group $(565.58 \pm 139.31)$ was significantly lower than the control group $(808.38 \pm 185.48)$, making it clear that intestinal IR promoted damage to the intestinal mucosa. On the other hand, villi height in the IR-PTX (790.35 \pm 171.52$)$, IPC-IR-SS (875.35 \pm 150.62$)$ and IPC-IR-PTX $(916.76 \pm 191.60)$ groups was significantly higher $(P=0.0088, P=0.0002, P<$ 0.0001 respectively) than that in the IR-SS group and had values similar to the standard values seen in the control group. In addition, villi height did not differ among the IR-PTX, IPCIR-SS and IPC-IR-PTX groups, indicating that the combination of IPC and PTX (IPC-IR-PTX group) did not differ significantly from these interventions alone (Figure 3). Crypt depth (Figure 4) was similar among the groups.

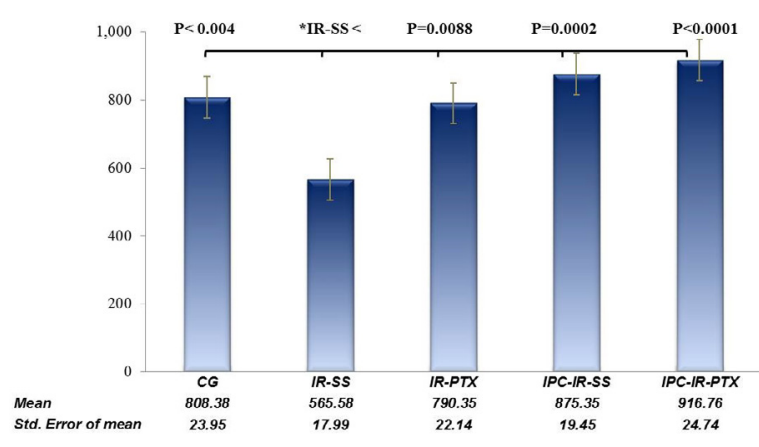

Figure 3 - Mean and standard error, and difference entre means groups the ANOVA OneWAY $(F=10.45, P<0.0001)$ the rat intestinal villi height measurements was different, and pos hoc Bonferroni test. $\left({ }^{*}\right)$ Analyze by pairs de difference statistically significant values was representing in the chart.

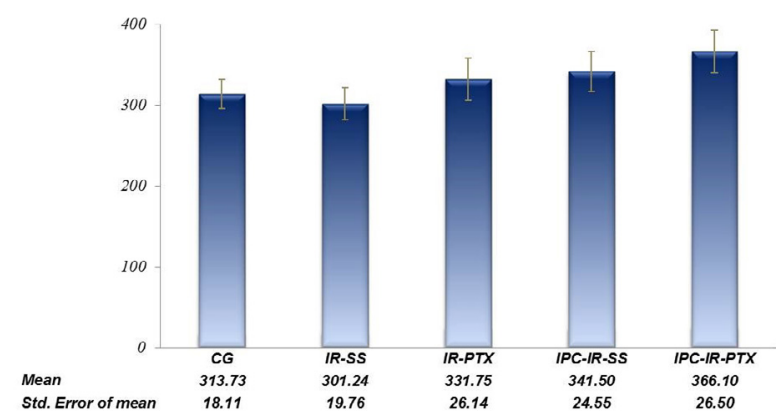

Figure 4 - Mean and standard error, 95\% confidence interval by factor and $F$ Test from the analysis of variance (ANOVA, $F=0.67$ ) was mayor than others groups $P>0.61$ of rat intestinal crypt depth measurements.

\section{- Discussion}

This study demonstrated that PTX or IPC can protect against ischemic damage in the small intestine in rats. Animals treated with PTX ( $80 \mathrm{mg} / \mathrm{kg}$ before ischemia) had high scores for mucosa preservation, high villi height and similar crypt depth to the IR group. Similarly, 
IPC (5 minutes of ischemia and 5 minutes reperfusion before 30 minutes of sustained ischemia) also protected the mucosa and the anatomical structure of the villi. However, the combination of both did not show an additive effect. The results of the combination were the same as the results of each procedure used alone.

Several clinical conditions are associated with IR injuries in the intestine and relevant morphological changes were determined for those conditions ${ }^{6,9,16-18}$. Epithelial detachment of the villi was previously described in the small intestine of rats using IR periods of 60 $\min$ and $90 \mathrm{~min}^{9,18-20}$. The results obtained in the present study were in accordance with these earlier observations.

The histological analyses showed detachment of epithelial cells (progressively worsening from the base to the top of the villi), sub-epithelial edema, and mild changes in the morphological structure of the submucosa with leukocyte infiltration. The mucosa damage was partially abolished with PTX treatment, with the IPC procedure, and with both interventions.

It is known that villus height and crypt depth vary according to the small intestine segment ${ }^{4,9,20,21}$. We focused the sample collection on the $20 \mathrm{~cm}$ area away from the duodenum-jejunum junction. In CG, the villi maintained their normal structure with an elongated axis and the lining with the intact enterocytes. There was no inflammatory edema or leucocyte infiltration on the axis or detachment of enterocytes. In the intestinal lumen, no cellular debris was detected.

The morphological changes maintained a standard structural derangement that started from the distal portion of the villi to the proximal portion, which was evident in the IRSS group. The greatest intensity of the lesion led to detachment of the mucosa and the appearance of debris in the intestinal lumen.

The height of villi varies within each intestinal region ${ }^{5,6,9,20,21}$. In animals subjected only to the IR procedure, the villous height was lower, indicating tissue damage. In the IR-PTX group, we found that the villi were well preserved, the leukocytes were less conspicuous, and the capillaries were dilated. Corroborating our findings, previous reports described more severe villous lesions in animals subjected to IR without any treatment compared to animals subjected to PTX treatment ${ }^{13,17,18,22-25}$.

Other reports using PTX at concentrations less than or equal to the concentrations used in this study have reported the preservation of the villi in the intestinal epithelium, similar to the results described here $^{13,17,18,22-26}$.

PTX showed a favorable effect by decreasing the score of the degree of cell damage. The height of the villi and the depth of the crypts were also preserved. The discovery that PTX had an anti-TNF- $\alpha$ effect stimulated its application into organ ischemia ${ }^{13,14,19,21-23}$. PTX is a nonselective phosphodiesterase inhibitor ${ }^{12,14,18,19}$ that decreases TNF-alpha and NFkB gene transcription ${ }^{13,14,19}$, affecting multiple steps in the cytokine/chemokine pathways and exerting beneficial immunomodulatory effects on inflammatory conditions directly and indirectly ${ }^{12,13,21-27}$. PTX increased the levels of specific cytokines, increased cyclic adenosine monophosphate (CAMP) levels and decreased TNF-alpha levels, resulting in anti-inflammatory and antioxidant properties ${ }^{12,18,21,23,25,26}$. These biochemical steps may account for the observed decrease in tissue damage. Although the focus of this study was not to investigate the mode of action of PTX, several reports have shown that its effects are favorable within the model proposed here $\mathrm{e}^{14,21,22,24-29}$.

IPC is a strategy by which tissues are subjected to controlled periods of ischemia and reperfusion before prolonged IR injury and is considered a ubiquitous phenomenon 
in mammals. The actual mechanism by which an episode of brief ischemia and reperfusion in an organ or tissue exerts protection against a subsequent sustained ischemia-reperfusion injury in a remote organ or tissue is currently unclear $^{3-11,16,30}$. In this research, the IPC procedures effectively protected the epithelial tissue and resulted in minimal smooth apical damage, minimal villous edema, and mildly dilated capillaries. The morphological characteristics of the IPC-treated group were similar to the control group. The microscopic structure was preserved as seen in the villous height and the depth of the crypts.

Although PTX and IPC separately elicited favorable results, the combination of these two treatments did not have a synergistic effect. Despite sharing some pathways, a possible explanation of this result may be the fact that PTX and IPC could also act through distinct signaling pathways, or at different time points, in the activation of factors that lead to protection against intestinal ischemia/ reperfusion. Future studies will need to address this issue.

\section{Conclusions}

In a model of short-term evaluation of intestinal ischemia and reperfusion, pentoxifylline and ischemic preconditioning had protective effects against mucosal injury. Although both may share common pathways, the simultaneous action of both did not provide better protection than the employment of each one separately.

\section{References}

1. Eltzschig HK, Eckle T. Ischemia and reperfusion-from mechanism to translation. Nat Med. 2011;17(11):1391-401. doi: 10.1038/nm.2507.

2. Granger DN, Kvietys PR. Reperfusion injury and reactive oxygen species: the evolution of a concept. Redox Biol. 2015;6:524-51. doi: 10.1016/j.redox.2015.08.020.

3. Camara-Lemarroy CR. Remote ischemic preconditioning as treatment for nonischemic gastrointestinal disorders: beyond ischemia-reperfusion injury. World J Gastroenterol. 2014;20(13):3572-81. doi: 10.3748/wjg.v20.i13.3572.

4. Mallick IH, Yang W, Winslet MC, Seifalian AM. Ischemia-reperfusion injury of the intestine and protective strategies against injury. Dig Dis Sci. 2004;49(9):1359-77. doi: 10.1023/B:DDAS.0000042232.98927.91.

5. Camprodon RA, Bowles MJ, Pockley AG, de Oca J. Anti-inflammatory effects of ischemic preconditioning on rat small bowel allografts. Transplant Proc. 2014;46(6):2146-9. doi: 10.1016/j.transproceed.2014.06.038.

6. SileriP,Sica G, Gentileschi P, Venza M, Manzelli A, Palmieri G, Spagnoli LG, Testa G, Benedetti $E$, Gaspari AL. Ischemic preconditioning protects intestine from prolonged ischemia. Transplant Proc. 2004;36(2):283-5. doi: 10.1016/j.transproceed.2004.01.078.

7. Bjornsson $B$, Winbladh A, Bojmar $L$, Sundqvist T, Gullstrand P, Sandstrom P. Conventional, but not remote ischemic preconditioning, reduces iNOS transcription in liver ischemia/reperfusion. World J Gastroenterol. 2014;20(28):9506-12. doi: 10.3748/wjg.v20.i28.9506.

8. Sola A, De Oca J, Gonzalez R, Prats N, RoselloCatafau J, Gelpi E, Jaurrieta E, Hotter G. Protective effect of ischemic preconditioning on cold preservation and reperfusion injury associated with rat intestinal transplantation. Ann Surg. 2001;234(1):98106. doi: $10.1097 / 00000658-200107000-$ 00015.

9. Takeshita M, Tani T, Harada S, Hayashi $H$, Itoh $\mathrm{H}$, Tajima $\mathrm{H}$, Ohnishi I, Takamura $\mathrm{H}$, Fushida S, Kayahara M. Role of transcription factors in small intestinal ischemiareperfusion injury and tolerance induced by ischemic preconditioning. Transplant Proc. 2010;42(9):3406-13. doi: 10.1016/j. transproceed.2010.06.038.

10.Taha MO, Miranda-Ferreira R, Chang AC, Rodrigues AM, Fonseca IS, Toral LB, Cardoso MR, Simoes MJ, Oliveira-Junior IS, Monteiro HP, Fagundes DJ, Taha NS, Caricati-Neto A. Effect of ischemic preconditioning on injuries caused by ischemia and reperfusion in rat 
intestine. Transplant Proc. 2012;44(8):23048. doi: 10.1016/j.transproceed.2012.07.056.

11. Hausenloy DJ, Yellon DM. Ischaemic conditioning and reperfusion injury. Nat Rev Cardiol. 2016;13(4):193-209. doi: 10.1038/ nrcardio.2016.5.

12.Yan K, Gao LN, Cui YL, Zhang Y, Zhou X. The cyclic AMP signaling pathway: exploring targets for successful drug discovery (Review). Mol Med Rep. 2016;13(5):371523. doi: $10.3892 / \mathrm{mmr} .2016 .5005$.

13.Ji $Q$, Zhang $L$, Jia $H$, Xu J. Pentoxifylline inhibits endotoxin-induced NF-kappa B activation and associated production of proinflammatory cytokines. Ann Clin Lab Sci. 2004;34(4):427-36. PMID: 15648785.

14.McCarty MF, O'Keefe JH, DiNicolantonio JJ. Pentoxifylline for vascular health: a brief review of the literature. Open Heart. 2016;3(1):e000365. doi: 10.1136/ openhrt-2015-000365.

15.Chiu CJ, McArdle AH, Brown R, Scott HJ, Gurd FN. Intestinal mucosal lesion in low-flow states. I. A morphological, hemodynamic, and metabolic reappraisal. Arch Surg. 1970;101(4):478-83. doi: 10.1001/ archsurg.1970.01340280030009.

16.Ji YY, Wang ZD, Wang SF, Wang BT, Yang $Z A$, Zhou XR, Lei NN, Yue WN. Ischemic preconditioning ameliorates intestinal injury induced by ischemia-reperfusion in rats. World J Gastroenterol. 2015;21(26):8081-8. doi: 10.3748/wjg.v21.i26.8081.

17.Savas C, Aras T, Cakmak M, Bilgehan A, Ataoglu O, Turkozkan N, Ozguner F, Yucesan $\mathrm{S}$, Dindar H. Pentoxifylline inhibits overflow and reduces intestinal reperfusion injury. J Pediatr Surg. 1997;32(6):905-10. doi: 10.1016/S0022-3468(97)90648-5.

18. Mustafa NA, Yandi M, Albayrak L, Yildiz K. Effect of pentoxifylline on the ischemiareperfusion injury of the intestine. Int Surg. 1995;80(2):152-5. PMID:8530233.

19.Zhang M, Xu YJ, Saini HK, Turan B, Liu PP, Dhalla NS. Pentoxifylline attenuates cardiac dysfunction and reduces TNF-alpha level in ischemic-reperfused heart. Am J Physiol Heart Circ Physiol. 2005;289(2):H832-9. doi: 10.1152/ajpheart.00178.2005.

20.Guan Y, Worrell RT, Pritts TA, Montrose $\mathrm{MH}$. Intestinal ischemia-reperfusion injury: reversible and irreversible damage imaged in vivo. Am J Physiol Gastrointest Liver
Physiol. 2009;297(1):G187-96. doi: 10.1152/ ajpgi.90595.2008.

21. Costantini TW, Loomis WH, Putnam JG, Kroll L, Eliceiri BP, Baird A, Bansal V, Coimbra R. Pentoxifylline modulates intestinal tight junction signaling after burn injury: effects on myosin light chain kinase. J Trauma. 2009;66(1):17-24. doi: 10.1097/ TA.0b013e318191bb1f.

22.Yang Q, Zheng FP, Zhan YS, Tao J, Tan SW, Liu HL, Wu B. Tumor necrosis factoralpha mediates JNK activation response to intestinal ischemia-reperfusion injury. World J Gastroenterol. 2013;19(30):492534. doi: 10.3748/wjg.v19.i30.4925.

23. Marques GM, Rasslan $R$, Belon $A R$, Carvalho JG, Neto RF, Rasslan S, Utiyama EM, Montero EF. Pentoxifylline associated to hypertonic saline solution attenuates inflammatory process and apoptosis after intestinal ischemia/reperfusion in rats. Acta Cir Bras. 2014;29(11):735-41. doi: 10.1590/S010286502014001800007.

24. Brasileiro JL, Inoye CM, Aydos RD, Silva IS, Falcao GR, Marks G, Pereira DM. Ischemia and reperfusion of rat small intestine using pentoxyfilline and prostaglandin E1. Acta Cir Bras. 2013;28(11):767-73. doi: 10.1590/ S012-86502013001100004.

25.Carsi JML, Lapena DC, Toledo AH, Fernandez CZ, Pereyra LHT. Pentoxifylline protects the small intestine after severe ischemia and reperfusion. Exp Clin Transplant. 2013;11(3):250-8. doi: 10.6002/ ect.2012.0222.

26. Rasslan R, Utiyama EM, Marques GM, Ferreira TC, da Costa VA, de Victo NC, Rasslan S, Montero EF. Inflammatory activity modulation by hypertonic saline and pentoxifylline in a rat model of strangulated closed loop small bowel obstruction. Int J Surg. 2014;12(6):594-600. doi: 10.1016/j. ijsu.2014.04.007.

27. Kreth S, Ledderose C, Luchting B, Weis F, Thiel M. Immunomodulatory properties of pentoxifylline are mediated via adenosinedependent pathways. Shock. 2010;34(1):106. doi: 10.1097/SHK.0b013e3181cdc3e2.

28.Sandoval J, Escobar J, Pereda J, Sacilotto N, Rodriguez JL, Sabater L, Aparisi L, Franco L, Lopez-Rodas G, Sastre J. Pentoxifylline prevents loss of PP2A phosphatase activity and recruitment of histone 
acetyltransferases to proinflammatory genes in acute pancreatitis. J Pharmacol Exp Ther. 2009;331(2):609-17. doi: 10.1124/ jpet.109.157537.

29.Fantin M, Quintieri L, Kusz E, Kis E, Glavinas $H$, Floreani $M$, Padrini R, Duda E, Vizler C. Pentoxifylline and its major oxidative metabolites exhibit different pharmacological properties. Eur J Pharmacol. 2006;535(1-3):301-9. doi: 10.1016/j.ejphar.2006.02.017.

30.Wang Z, Ji Y, Wang S, Wang R, Li Z, Kang A, $\mathrm{Xu} \mathrm{H}$, Shi M, Zhao M. Protective effect of intestinal ischemic preconditioning on ischemia reperfusion-caused lung injury in rats. Inflammation. 2015;38(1):424-32. doi: 10.1007/s10753-014-0047-3.

\section{Acknowledgement}

To Prof. Manuel de Jesus Simões, Chairman of Division of Histology and Structural Biology, Department of Morphology and Genetic, Universidade Federal de São Paulo (UNIFESP).

\section{Correspondence:}

Profa Teresinha Regina Ribeiro de Oliveira Rua João Vicente Ferreira, 1789

79825050 Dourados - MS Brasil

Tels.: (55 67)3421-9456 / 99296-3030

gfo1oliveira@gmail.com

oliveiratregina@gmail.com

Received: Mar 06, 2017

Review: May 05, 2017

Accepted: June 09, 2017
Conflict of interest: none

Financial source: UFGD, Ministério da Educação do Brasil
${ }^{1}$ Research performed at Division of Surgical Techniques and Experimental Surgery, Department of Surgery, Universidade Federal de São Paulo (UNIFESP), Brazil. 